\title{
Mindfulness-based cognitive therapy as an alternative to maintenance antidepressant medication to prevent relapse and recurrence in depression
}

\author{
Marloes Huijbers, Anne Speckens \\ Department of Psychiatry, Radboud University Medical Center, Nijmegen, The Netherlands \\ Correspondence to Marloes Huijbers, marloes.huijbers@radboudumc.nl
}

ABSTRACT FROM: Kuyken W, Hayes R, Barrett B, et al. Effectiveness and cost-effectiveness of mindfulness-based cognitive therapy compared with maintenance antidepressant treatment in the prevention of depressive relapse or recurrence (PREVENT): a randomised controlled trial. Lancet 2015;386:63-73.

\section{WHAT IS ALREADY KNOWN ON THIS TOPIC}

Patients with major depressive disorder have a high risk of relapse/ recurrence. ${ }^{1}$ Maintenance antidepressant medication (mADM) is typically recommended to prevent relapse/recurrence, but many individuals prefer psychosocial interventions. ${ }^{2}$ Previous studies have indicated that mindfulness-based cognitive therapy with taper support (MBCT-TS) may be at least as effective as mADM in reducing the risk of depressive relapse/recurrence, ${ }^{3}$ and the aim of the current study was to compare these interventions in a definitive trial.

\section{METHODS OF THE STUDY}

In this 24-month single-blind, randomised controlled trial, 424 adult patients with three or more previous episodes of depression, who were using mADM, were included. They were recruited between March 2010 and October 2011, from 95 primary care practices in the UK. Participants were randomly allocated to receive either MBCT-TS or mADM lactive comparator). MBCT-TS consisted of an 8-week group intervention designed to help patients prevent depressive relapse/recurrence by developing non-judgemental awareness of their automatic patterns of thinking and behaviour. MBCT-TS fosters an intentional, skilful response to difficult experiences. Support to taper or discontinue mADM was included in the programme. By contrast, the mADM condition consisted of support from patients' general practitioners to continue their medication use over the course of the study period.

Primary outcome was time to relapse or recurrence of a depressive episode as measured with the Structured Clinical Interview for Diagnostic and Statistical Manual of Mental Disorders, 4th Edition, disorders. Secondary outcomes were number of depression-free days, residual depressive symptoms, psychiatric and medical comorbidity, quality of life and costeffectiveness. Only assessors were blinded to treatment allocation.

\section{WHAT THIS PAPER ADDS}

- Time to relapse or recurrence of depression over 24 months did not differ between patients in the MBCT-TS group and those in the mADM group (HR 0.89, 95\% Cl 0.67 to 1.18). A total of 94 (44\%) of 212 patients in the MBCT group relapsed, compared with 100 $(47 \%)$ of 212 in the mADM.

- MBCT-TS was no better than mADM for the number of depressionfree days, residual depressive symptoms, psychiatric and medical comorbidity, and quality of life.

- In total, ten adverse events were reported. In each group, there were three non-fatal and two fatal serious adverse events. No adverse events were deemed related to the interventions or the trial.

- MBCT-TS was not more cost-effective than mADM in terms of either relapse/recurrence or quality of life.

- Subgroup analyses indicated that patients who reported severe childhood abuse benefited more from MBCT-TS than from mADM in terms of relapse/recurrence risk (HR 0.53, 95\% Cl 0.29 to 0.95), which supports the idea that MBCT may be most effective for patients with increased vulnerability due to childhood trauma. ${ }^{4}$

\section{LIMITATIONS}

- The results of the current study may only be generalisable to patients who are willing to accept both types of treatment.

- Owing to the pragmatic nature of the trial, a subgroup of participants allocated to the MBCT-TS group either did not reduce their mADM (13\%) or did not discontinue completely (17\%). This may have inflated the effectiveness of MBCT-TS, as the combination of MBCT and mADM is possibly more effective than MBCT-TS. ${ }^{5}$

- Results might be biased by the fact that patients and clinicians were inevitably aware of treatment allocation, and the absence of an attentional control group means that any effects of MBCT-TS may not be specific to this intervention.

- The established competency of the four trial therapists may have contributed to the effectiveness of MBCT to an extent that may be difficult to attain when MBCT is delivered in routine clinical practice.

\section{WHAT NEXT IN RESEARCH?}

In addition to examining MBCT as a possible alternative to mADM, it might be interesting to explore to what extent and for which patients the combination of MBCT and mADM might be the better option. There is also a need for more information about the effectiveness of MBCT in secondary and tertiary care settings with higher levels of psychiatric and/or somatic comorbidity.

\section{DO THESE RESULTS CHANGE YOUR PRACTICES AND WHY?}

Yes. This study is the largest trial of mindfulness-based approaches to date, providing evidence to support MBCT-TS as an alternative to mADM. The trial had a sound methodology including high treatment fidelity, good adherence rates, masked outcome assessment and a relatively long follow-up. Thus, we would certainly recommend MBCT-TS to patients who want to taper their medication.

Competing interests None declared.

doi:10.1136/eb-2015-102148

Received 12 June 2015; Revised 16 August 2015; Accepted 21 August 2015

\section{REFERENCES}

1. Richards D. Prevalence and clinical course of depression: a review. Clin Psychol Rev 2011;31:1117-25.

2. van Schaik D, Klijn A, van Hout $H$, et al. Patients' preferences in the treatment of depressive disorder in primary care. Gen Hosp Psychiat 2004;26:184-9.

3. Segal ZV, Bieling P, Young T, et al. Antidepressant monotherapy vs sequential pharmacotherapy and mindfulness-based cognitive therapy, or placebo, for relapse prophylaxis in recurrent depression. Arch Gen Psychiatry 2010;67:1256-64.

4. Williams JMG, Crane C, Barnhofer T, et al. Mindfulness-based cognitive therapy for preventing relapse in recurrent depression: a randomized dismantling trial. $J$ Consult Clin Psychol 2014;82:275-86.

5. Huijbers MJ, Spinhoven P, Spijker J, et al. Discontinuation of antidepressant medication after mindfulness-based cognitive therapy for recurrent depression: randomised controlled non-inferiority trial. Br J Psychiat (in press). 